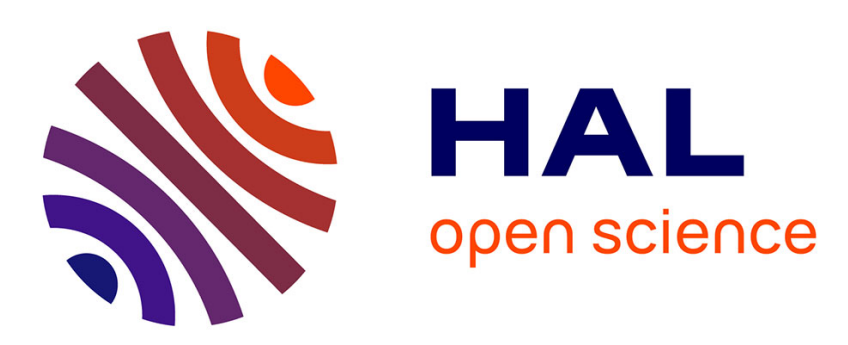

\title{
Sensing Mechanism of Surface Acoustic Wave Magnetic Field Sensors Based on Ferromagnetic Films
}

Yang Yang, Harshad Mishra, Tao Han, Sami Hage-Ali, Michel Hehn, Omar Elmazria

\section{- To cite this version:}

Yang Yang, Harshad Mishra, Tao Han, Sami Hage-Ali, Michel Hehn, et al.. Sensing Mechanism of Surface Acoustic Wave Magnetic Field Sensors Based on Ferromagnetic Films. IEEE Transactions on Ultrasonics, Ferroelectrics and Frequency Control, 2021, 10.1109/tuffc.2021.3069382 . hal-03239335

\section{HAL Id: hal-03239335 \\ https://hal.science/hal-03239335}

Submitted on 27 May 2021

HAL is a multi-disciplinary open access archive for the deposit and dissemination of scientific research documents, whether they are published or not. The documents may come from teaching and research institutions in France or abroad, or from public or private research centers.
L'archive ouverte pluridisciplinaire HAL, est destinée au dépôt et à la diffusion de documents scientifiques de niveau recherche, publiés ou non, émanant des établissements d'enseignement et de recherche français ou étrangers, des laboratoires publics ou privés. 


\title{
Sensing Mechanism of Surface Acoustic Wave Magnetic Field Sensors Based on Ferromagnetic Films
}

\author{
Yang Yang, Student Member, IEEE, Harshad Mishra, Member, IEEE, Tao Han*, Member, IEEE, Sami Hage-Ali, \\ Member, IEEE, Michel Hehn, and Omar Elmazria*, Senior Member, IEEE
}

\begin{abstract}
Surface acoustic wave (SAW) sensors with ferromagnetic materials are used to measure magnetic fields or electric currents. The magnetic field sensitivities of SAW magnetic field sensors are essentially influenced by various factors. The sensing mechanism is complex due to the multiphysics coupling of the magnetic field, solid mechanics, and electric field. The magnetostriction effect, $\Delta \mathbf{E}$ effect, and the third-order material constants are taken into consideration. The shape demagnetizing effect is reduced by increasing the lengthwidth ratio and length-height ratio of a ferromagnetic film on a SAW resonator. The model is verified by experiments and accurately predicts the magnetic field sensitivities of SAW resonant magnetic field sensors. The factors affecting the sensitivities are investigated from the perspective of the sensing mechanism. A grooved sensing surface structure is explored for an improved sensitivity. The results are beneficial to design reliable SAW magnetic field sensors with an enhanced sensitivity.
\end{abstract}

Index Terms - Surface acoustic wave, magnetic field, sensing mechanism.

\section{INTRODUCTION}

$\mathrm{M}$ AGNETIC fields are generally measured in scientific researches and engineering applications. Magnetic field sensors, such as Hall sensors, anisotropic magnetoresistive sensors, giant magnetoresistive sensors, and fluxgate sensors, need power supply that restricts their practical applications [1], [2]. Wireless and passive surface acoustic wave (SAW) sensors are popularly used in industrial applications for detections, monitoring, and measurements [3]. With the rapid and wide developments of ferromagnetic materials in the fields of sensors, transducers, oscillators, and actuators [4], SAW devices based on ferromagnetic films have been attracted extensive interest in the developments of wireless and passive magnetic field or electric current sensors [5]-[7]. The sensitivities of SAW magnetic field devices have been investigated using different ferromagnetic materials [8]-[13]. A sensitivity of $-6.75 \mathrm{ppm} / \mathrm{mT}$ is achieved on a $\mathrm{TbFe}_{2} / \mathrm{LiNbO}_{3}$ structure [9]. A SAW velocity change of $310 \mathrm{ppm}$ is attained using a $\mathrm{CoCr}$ film [10]. Magnetostrictive $\mathrm{FeCo}$ films are

Manuscript received, 2021. This work was supported in part by the National Natural Science Foundation of China (under Grant No. U1837210, No.11774230), in part by the French PIA project "Lorraine Université d'Excellence" (ANR-15-IDEX-04-LUE), and in part by ANR JCJC SAWGOOD (ANR-18-CE42-0004-01). (*Corresponding authors: Tao Han; Omar Elmazria.)

Yang Yang, and Tao Han are with the School of Electronic Information and Electrical Engineering, Shanghai Jiao Tong University, Shanghai, 200240, China. (e-mail: yangyang2015@sjtu.edu.cn; than@ @stu.edu.cn)

Harshad Mishra, Sami Hage-Ali, Michel Hehn, and Omar Elmazria are with the Institute Jean Lamour UMR 7198, Universite de Lorraine - CNRS, Nancy, 54000, France. (e-mail: omar.elmazria@univ-lorraine.fr) deposited on SAW sensors for magnetic field and electric current measurements [11]. A SAW sensor based on a FeGa film obtains a relative velocity change of $0.64 \%$ [12]. The frequency responses and bandwidths of SAW delay line magnetic field/ electric current sensors based on $\mathrm{FeCoSiB}$ are studied [13].

As stated in these researches, properties of ferromagnetic materials have crucial influences on the sensitivities. The magneto-mechanical coupling sensitive effect contained in ferromagnetic films can be combined with SAW technology through the mechanical-electro coupling. Using a ferromagnetic film with both of $\Delta \mathrm{E}$ effect and magnetostriction effect, such as $\mathrm{CoFeB}$, is beneficial for the sensitivity improvement. Considering that $\Delta \mathrm{E}$ effect and magnetostriction effect are coupled with each other, it is potential that the contributions of $\Delta \mathrm{E}$ effect and magnetostriction effect on sensitivities can be superimposed under certain multi-physics coupling conditions. Therefore, a multi-physics coupling model for SAW magnetic field sensors with complex boundary conditions need to be established. Sinha and Tiersten have developed a perturbation theory for small acoustic fields superposed on a bias to calculate the behavior of surface waves [14]. Considering that the vibration frequencies of biasing fields, such as the magnetic field and thermal field, are far lower than that of the incremental field caused by SAW, dynamic characteristics of biasing fields and SAW are hence processed separately. This paper studies a SAW multi-physics coupling model to investigate the inherent sensing mechanism of SAW magnetic field sensors according to Tiersten's perturbation theory [15]. The influences of magnetic fields on SAW are transformed into the form of stresses and strains in the model. The nonlinear piezoelectric constitutive equations with perturbation terms in multi-physics fields are established and solved by weak form equations [16].

Two types of sensitive structures, where the ferromagnetic material is either patterned as electrodes or used as a full film, are discussed using the multi-physics coupling model to explore methods for magnetic field sensitivity improvement. The simulation results are compared with experimental results from the literature, including our previous work. The factors affecting the sensitivities are investigated. It is found that the saturation magnetic flux density influences the measurement range and the sensitivity. The reduced saturation magnetic flux density of a soft magnetic film is beneficial to increase the magnetic field sensitivity. Furthermore, the shapes of ferromagnetic electrodes contribute to the magnetizations and sensitivities. The impact of shape demagnetizing effect on 
sensor performance is studied. The full ferromagnetic film SAW magnetic field sensor structure has the advantage of physically reducing shape demagnetizing fields by increasing the length-width ratio and length-height ratio. More SAW energy concentrated in the ferromagnetic film also makes a contribution to improving the sensitivity. A grooved sensing surface structure is studied for an improved sensitivity due to enhanced sensing areas and magnetostrictive displacement deformations.

The remaining of this paper is organized as follows: in section II, a SAW multi-physics coupling model and the sensing mechanism of SAW magnetic field sensors are investigated. In section III, the multi-physics coupling model is verified by experiments, and the contributions of various factors towards sensitivities of SAW magnetic field sensors are studied. Conclusions are discussed in section IV.

\section{SENSING MECHANISM ANALYSIS}

A SAW magnetic field sensor based on a ferromagnetic film is composed of a piezoelectric substrate, interdigital transducers (IDT), an isolating layer, and a ferromagnetic film, as indicated in Fig. 1(a). The wave propagation is affected by several factors in a SAW magnetic field sensor. Table. I lists the descriptions of the magnetostriction and $\Delta \mathrm{E}$ effect. These properties of ferromagnetic films are taken into the multiphysics coupling model to study the sensing mechanism and to calculate the magnetic field sensitivity characteristics.

TABLE. I INHERENT PROPERTIES OF FERROMAGNETIC FILMS

\begin{tabular}{c|c}
\hline Properties & Descriptions \\
\hline Magnetostriction & $\begin{array}{c}\text { Magnetic field dependent magnetostriction- } \\
\text { induced strains } \\
\text { Inverse magnetostriction } \\
\Delta \mathrm{E} \text { effect }\end{array}$ \\
$\begin{array}{c}\text { Magnetizations affected by applied stresses } \\
\text { Magnetic field dependent elastic constants }\end{array}$ \\
\hline
\end{tabular}

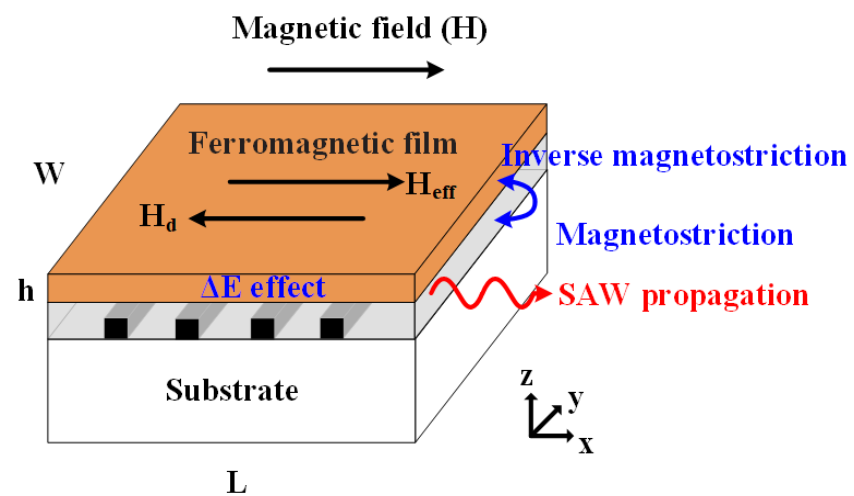

(a)

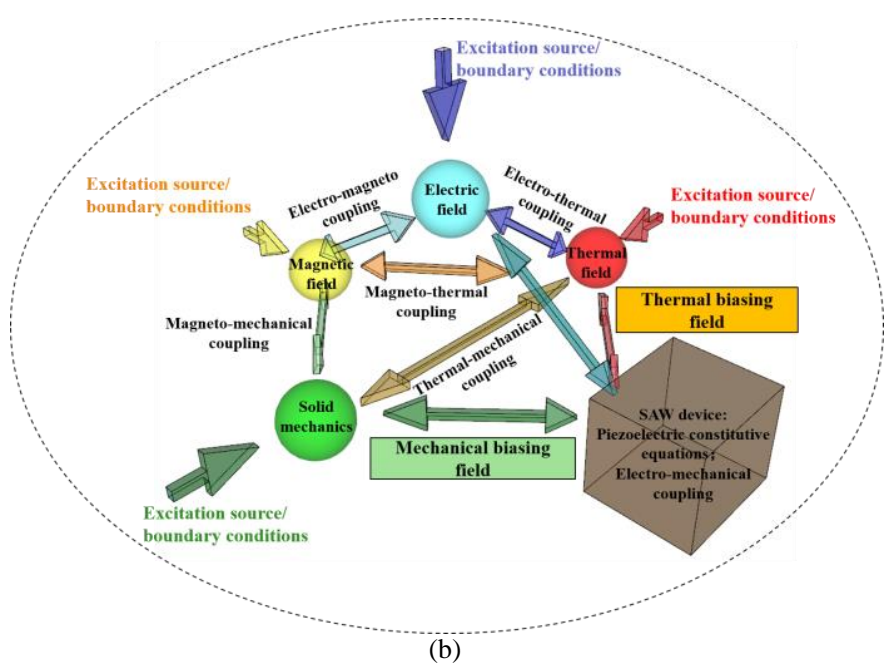

Fig. 1. (a) The diagram of a ferromagnetic film based SAW resonator structure (full film configuration) and the interaction between the ferromagnetic film and SAW. (b) Multi-physics coupling relationships for SAW magnetic field sensors.

SAW magnetic field sensors are electro/magneto/solid mechanical/thermal multi-physics coupling systems. The sensing mechanism of SAW sensor is investigated from the perspective of multi-physics coupling. The electro/magneto/solid mechanical/thermal multi-physics coupling relationships for SAW magnetic field sensors are illustrated in Fig. 1(b). The piezoelectric constitutive equations include the direct coupling of electric and solid mechanical fields. The multi-physics coupling equations are a combination of the piezoelectric coupling, magneto-mechanical coupling, magneto-thermal coupling, electro-magneto coupling and thermal-mechanical coupling.

From the perspective of sensing mechanism, the magnetic field affects the solid mechanics directly through ferromagnetic films and then influences SAW indirectly. The direct coupling of magneto-piezoelectric equations requires effective material constants with unknown values that increase the uncertainty of accuracy and complexity of computations. Therefore, the magnetopiezoelectric direct coupling is simplified to a weak coupling of magnetic and piezoelectric fields according to the physical mechanisms. The electric and magnetic fields are coupled by Maxwell equations. Because the velocity of SAW in piezoelectric materials is $10^{-5}$ times lower than that of electromagnetic waves, the coupling between the electromagnetic wave and acoustic wave is weak. Therefore, the quasi-static approximation and electrostatic field are involved in the piezoelectric coupling. The impact of the SAW induced electrostatic charge on the magnetic field is neglected to simplify the model.

In SAW sensing applications, such as temperature and pressure sensing, the thermal and mechanical fields work as biasing fields influencing SAW velocities. The sensing mechanism of SAW sensors is prominently determined by the biasing fields. The behavior of SAW under biasing fields is described by nonlinear piezoelectric constitutive equations for infinitesimal incremental fields superposed on a finite bias. 
Assuming that the frequency of the small dynamic perturbations caused by the magnetic field is much lower than the SAW frequency, the magnetic field is considered as an increment biasing field. A combination of direct coupling and sequential coupling is established between the magnetic field and piezoelectric field based on Tiersten's nonlinear perturbation theory in the Lagrangian description [15]. SAW wave motion equations in multi-physics fields are defined as follows:

$$
\left\{\begin{array}{l}
\nabla \cdot \boldsymbol{K}+\rho_{0} \boldsymbol{f}=\rho_{0} \frac{\partial^{2} \boldsymbol{u}}{\partial t^{2}} \\
\nabla \cdot \boldsymbol{D}=\rho_{e}
\end{array}\right.
$$

where $\boldsymbol{K}, \boldsymbol{f}$, and $\boldsymbol{D}$ represent the stress tensor, mechanical body force, and the electric displacement, respectively. $\boldsymbol{u}, \rho_{0}$, and $\rho_{e}$ are the displacement, mass density, and free charge density, respectively. The nonlinear piezoelectric constitutive equations with perturbation terms in multi-physics fields are defined as follows:

$$
\left\{\begin{array}{l}
K_{L \gamma}=G_{L \gamma J \alpha}\left(T^{H}, S^{H}, w^{H}, \Theta\right) u_{\alpha, J}-R_{J L \gamma}\left(S^{H}, w^{H}, E^{0}, \Theta\right) E_{J} \\
D_{L}=R_{L J \alpha}\left(S^{H}, w^{H}, E^{0}, \Theta\right) u_{\alpha, J}+N_{L J}\left(S^{H}, E^{0}, \Theta\right) E_{J}
\end{array}\right.
$$

where $G_{L \gamma J \alpha}, R_{L J \alpha}, N_{L J}$ are the effective elastic constants, effective piezoelectric constants, and effective dielectric constants as a function of the magnetostrictive stress tensor $T^{H}$, magnetostrictive strain tensor $S^{H}$, the deformation gradients $w^{H}$ generated by the magnetostriction effect, the initial electric field $E^{0}$, and the temperature $\Theta$, respectively. The magnetostrictive stress tensor $T^{H}$ is imported into the effective elastic constants with Tiersten's perturbation method [15]. These parameters ultimately influence the SAW propagation indirectly. Sequentially, the resonance frequency of the SAW magnetic field sensor is influenced. The third-order elastic constants, third-order piezoelectric constants, and third-order dielectric constants are imported into the effective material constants as nonlinear contributions. Biasing magnetostrictive strains and geometry deformations are inhomogeneous. Effective material constants are functions of the biasing fields and are therefore various at different mesh nodes. The nonlinear piezoelectric constitutive equations are accurately solved using the finite element weak form method.

The interaction between the ferromagnetic film and SAW is realized by the bidirectional coupling of the magnetic field and solid mechanics, irrespective of the thermal exchange between the solid mechanics and magnetic field. The magnetostriction effect with nonlinear geometry deformations and $\Delta \mathrm{E}$ effect are added into the model where the magnetic field and the mechanical field are coupled. The ferromagnetic film converts the applied external magnetic field into mechanical perturbations. The magnetizations have direct impacts on the magnetostrictive strains. In many cases, deposited ferromagnetic films exhibit a uniaxial anisotropy due to deposition geometry or presence of magnetic fields. The magnetostriction-induced strain is defined as follows:

$$
S_{J \alpha}^{H}=\left\{\begin{array}{l}
\frac{\lambda_{s}}{M_{s}^{2}}\left[M_{J}^{2}-\frac{1}{2}\left(M_{k}^{2}+M_{l}^{2}\right)\right] \quad(J=\alpha \neq k \neq l) \\
\frac{3 \lambda_{s}}{2 M_{s}^{2}} M_{J} M_{\alpha} \quad(J \neq \alpha)
\end{array} \quad(J \neq \alpha)\right.
$$

where $\lambda_{s}$ and $M_{s}$ are the saturation magnetostrictive coefficient and saturation magnetization, respectively. The subscript symbols $J, \alpha, k, l=1,2,3$ that represent the directions. As the ferromagnetic film is magnetized, internal stresses are generated inside the film due to magneto- mechanical coupling. The effective elastic constants of a ferromagnetic film at an applied magnetic field include the second-order elastic constants, third-order elastic constants and $\Delta \mathrm{E}$ effect induced variations of the elastic constants $\Delta c_{\mathrm{ij}}$. Because the Rayleigh wave is a combination of longitudinal wave mode and shear vertical (SV) wave mode, and the Love wave is shear horizontal $(\mathrm{SH})$ wave mode, they are mostly sensitive to the variations of elastic constants $c_{11}, c_{44}$, and $c_{66}$. Previous studies have shown that the dependences of $\Delta c_{11}$ and $\Delta c_{66}$ are caused by the $\Delta \mathrm{E}$ effect when the applied magnetic field is along the hard magnetic axis [17], [18]. The $\Delta \mathrm{E}$ effect induced $\Delta c_{44}$ along the hard axis are defined as follows:

$$
\Delta c_{44}= \begin{cases}-\frac{\left(-3 \lambda_{s} c_{44}\right)^{2}\left(\frac{H^{2}}{H_{s}^{2}}\right)}{\mu_{0} M_{s}\left(H+H_{s}\left(1-\frac{H^{2}}{H_{s}^{2}}\right)+H_{m e}\right)} & \left(H \leq H_{s}\right) \\ -\frac{\left(-3 \lambda_{s} c_{44}\right)^{2}}{\mu_{0} M_{s}\left(H+H_{m e}\right)} & \left(H>H_{s}\right)\end{cases}
$$

where $H_{s}$ represents the saturation magnetic flux density. $H_{m e}$ is the magneto-elastic field [17]. $c_{44}$ represents the secondorder elastic constant. $\mu_{0}$ is the vacuum permeability. Before the magnetization saturation, the contribution of $\Delta \mathrm{E}$ effect is the major effect on the variations of effective elastic constants and the sensing mechanism. The $\Delta \mathrm{E}$ effect gradually becomes weak as the magnetic field increases above the saturation magnetic flux density. The magnetostrictive coefficient and the saturation magnetization determine the maximum variations of elastic constants. The magnetostriction effect leads to the magnetostrictive strains in response to an externally applied magnetic field, and $\Delta \mathrm{E}$ effect causes the changes of the elastic constants. The two effects, that occur simultaneously, allow the transduction between magnetic and mechanical energies. Combined with Eq. (3), the magnetomechanical coupling equations with the magnetostriction and $\Delta \mathrm{E}$ effect are defined as follows:

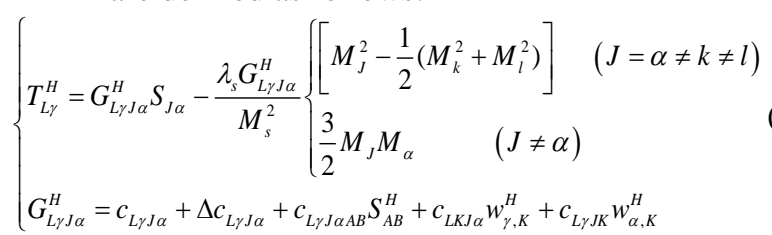

where $G_{L \gamma J \alpha}^{H}$ is the effective elastic constant of the ferromagnetic film. $c_{L \gamma J \alpha}$ and $c_{L \gamma J \alpha A B}$ are the second-order elastic constant and the third-order elastic constant, respectively [15]. If the magnetostrictive coefficient is negative, the contribution of magnetostriction effect will be the opposite of $\Delta \mathrm{E}$ effect on sensitivities when the magnetic 
field is below the saturation magnetic flux density. On the contrary, as the magnetostrictive coefficient is positive, the contributions of magnetostriction effect and $\Delta \mathrm{E}$ effect on sensitivities are superimposed in SAW propagation direction along the hard axis.

The magnetostriction effect and $\Delta \mathrm{E}$ effect influence the SAW propagation. In return, the SAW propagation impinges additional stresses on the ferromagnetic film and thus influences the magnetization of the ferromagnetic film due to the inverse magnetostriction effect. Therefore, the magnetization of the ferromagnetic film depends on the applied magnetic field and SAW induced stress. The nonlinear magnetization with demagnetizing effect of a ferromagnetic film along the hard axis is quantified by the Langevin function [19]:

$$
\left\{\begin{array}{l}
\boldsymbol{M}=M_{s}\left[\operatorname{coth}\left(\frac{3 \chi\left|\boldsymbol{H}_{e f f}\right|}{M_{s}}\right)+\frac{M_{s}}{3 \chi\left|\boldsymbol{H}_{\text {eff }}\right|}\right] \frac{\boldsymbol{H}_{\text {eff }}}{\left|\boldsymbol{H}_{\text {eff }}\right|} \\
\boldsymbol{H}_{\text {eff }}=\boldsymbol{H}+\frac{3 \lambda_{s}}{\mu_{0} M_{s}^{2}} \overline{\boldsymbol{T}} \boldsymbol{M}+\boldsymbol{H}_{\boldsymbol{d}}
\end{array}\right.
$$

where $\chi$ is the initial susceptibility. $\boldsymbol{H}_{\text {eff }}$ represents the effective magnetic field in the ferromagnetic film. $\boldsymbol{M}$ represents the magnetization. $\boldsymbol{H}$ is the applied external magnetic field. $\boldsymbol{H}_{\boldsymbol{d}}$ is the demagnetizing field. $\overline{\boldsymbol{T}}$ is the SAW induced stress tensor. The equations explain the transduction of the elastic stress to the magnetic state in the presence of stresses from the SAW propagation. The contributions of the external magnetic field, the magnetic field induced by stress, and the demagnetizing field are considered in the effective magnetic field. The combination of Eq. (5) and Eq. (6) realizes a bidirectional coupling of the magnetic field and solid mechanics.

With the magnetization process, the demagnetizing field appears inside the ferromagnetic film. The demagnetizing field is in the opposite direction of the magnetization:

$$
\boldsymbol{H}_{d}=-N_{d} \boldsymbol{M}
$$

where $N_{d}$ is a demagnetization factor that describes the strength of the demagnetizing field inside the film. Shape demagnetizing effects weaken the effective magnetic field and cause shape anisotropy. Assuming the ferromagnetic film is a cuboid with the size of length (L), width (W), and height (h), as shown in Fig. 1(a), the shape demagnetization factor along the center axis in the ferromagnetic film is calculated according to the method in Ref. [20].

In a ferromagnetic full film based SAW device configuration, the ferromagnetic film covers the areas of IDTs and reflection gratings. According to the size of a SAW magnetic resonator, the width of the ferromagnetic film approximates to the aperture and is much smaller than the length. We set the film length: $\mathrm{L}=4 \mathrm{~mm}$, width: $\mathrm{W}=0.5 \mathrm{~mm}$. Fig. 2 (a) and (b) show the shape demagnetization factor distributions of ferromagnetic film in the $\mathrm{x}$ and $\mathrm{y}$ directions versus film thicknesses. It is found that the shape demagnetization factor reaches the minimum at the center of the film. The shape demagnetization factor increases when the position is close to the film edge. $N_{d}$ is mainly within the range of $10^{-4}-10^{-6}$ in the $x$ direction that is much lower than that of
$10^{-3}-10^{-4}$ in the $y$ direction. Therefore, the shape demagnetizing field in the $\mathrm{x}$ direction is very weak inside the film and can be neglected. Fig. 2 (c) illustrates the dependence of $N_{d}$ on the length-width ratio and length-height ratio. The shape demagnetization factor in the $\mathrm{x}$ direction decreases with the increase of the length-width ratio and length-height ratio. The effective magnetic susceptibility increases correspondingly. A reduced thickness of the ferromagnetic film and an increased length-width ratio are beneficial for reducing the shape demagnetizing effect within the film plane.

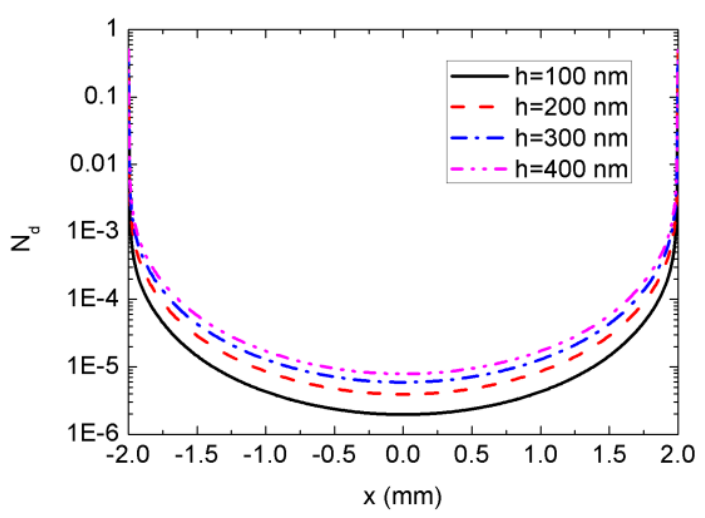

(a)

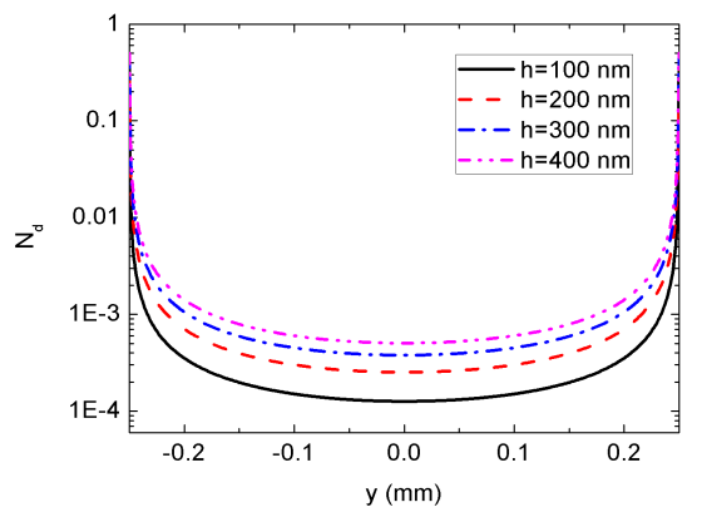

(b)

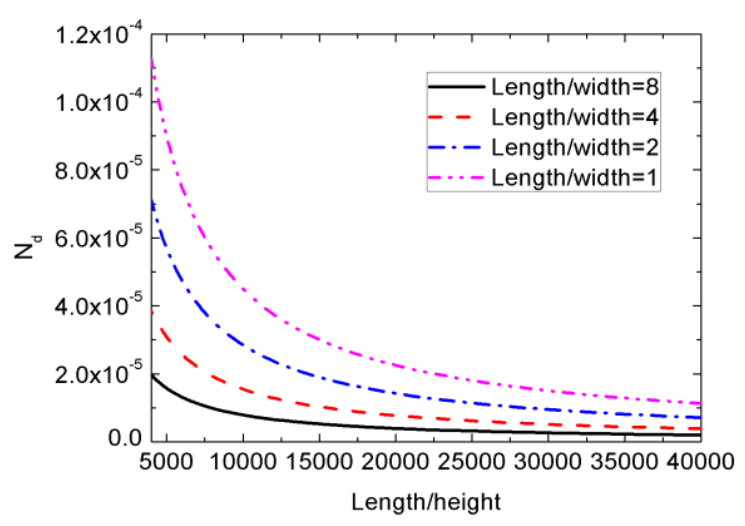

(c)

Fig. 2. Shape demagnetization factor distributions of a ferromagnetic film (a) in the $\mathrm{x}$ direction. (b) in the $\mathrm{y}$ direction. (c) the shape demagnetization factor variations in the $\mathrm{x}$ direction versus the length-width ratio and length-height ratio. 
The coupled electro/magneto/solid mechanical multiphysics equations are defined with the combination of wave motion equations Eq. (1), piezoelectric constitutive equations Eq. (2), the magneto-mechanical coupling equations with the magnetostriction and $\Delta \mathrm{E}$ effect Eq. (5), magnetization equations with demagnetizing effect Eq. (6), and Maxwell equations. In this way, the magnetic field are coupled with the SAW. The wave propagation properties and resonance frequency of SAW are solved based on the simultaneous solution of multi-physics equations. The measurement range is usually expressed as the magnetic field range from 0 to the magnetic field value of the magnetization saturation. The expected dependence of the calculated magnetic response on the frequency of the SAW mode is monotonous and nearly linear. The sensitivity is defined as the maximum slope of resonance frequency variations during the measurement range. Therefore, the saturation magnetic flux density is a significant factor that determines the measurement range and the sensitivity.

\section{RESULTS AND DISCUSSION}

The factors affecting the sensitivities are investigated from the perspective of the sensing mechanism in this part. Two configurations are considered: in the first, the ferromagnetic material serves as electrodes, and in the second, the ferromagnetic material is a full film on the top of a SAW structure. To verify the accuracy of the theoretical model, the simulation results are compared with the experimental ones extracted from literature, including our previous work.

\section{A. Ferromagnetic material as electrodes}

\section{1) $\mathrm{Ni} / 41^{\circ} \mathrm{YX}$-cut $\mathrm{LiNbO}_{3}$ structure}

Firstly, the magnetic field sensitivity of the Rayleigh wave mode on the $\mathrm{Ni}$ electrodes $/ 41^{\circ} \mathrm{YX}$-cut $\mathrm{LiNbO}_{3}$ resonator is calculated. The structure is shown in Fig. 3. The third-order material constants of $\mathrm{LiNbO}_{3}$ are taken from Ref. [21]. The material constants of $\mathrm{Ni}$ are as follows: $\rho_{0}=8900 \mathrm{~kg} / \mathrm{m}^{3}$, $c_{11}=224 \mathrm{GPa}, c_{12}=156 \mathrm{GPa}, c_{44}=120 \mathrm{GPa}, c_{111}=-2104 \mathrm{GPa}$, $c_{112}=-1345 \mathrm{GPa}, c_{123}=59 \mathrm{GPa}, c_{144}=-180 \mathrm{GPa}, c_{166}=-757 \mathrm{GPa}$, $c_{456}=-42 \mathrm{GPa}, \lambda_{s}=-40 \mathrm{ppm}, M_{s}=490 \mathrm{kA} / \mathrm{m}$ [22]. The shapes of the electrodes are built as cuboids in the electro/magneto/solid mechanical multi-physics coupling model. The heights and widths of electrodes are set using the finite element method. An external magnetic field is applied along the hard axis. The shapes of the electrodes contribute to the anisotropy. The magnetization of the Ni electrode changes with the electrode's height and width, by the virtue of shape induced anisotropy. Thus, the sensitivity of the device also changes accordingly. Fig. 4 illustrates the calculated magnetization of $\mathrm{Ni}$ electrodes with different metallization ratios (r). The thicknesses of $\mathrm{Ni}$ electrodes are $50 \mathrm{~nm}$. The wavelength is $6.5 \mu \mathrm{m}$. The anisotropy field decreases and the effective magnetic susceptibility increases when the metallization ratio increases from 0.4 to 0.6 . It is due to the varying shape demagnetizing field by expanding the widths of electrodes. Thus, the shapes of the electrodes also influence the sensitivity. Fig. 5 illustrates the calculated sensitivities of Rayleigh wave mode on $\mathrm{Ni}$ electrodes $/ 41^{\circ} \mathrm{YX}$-cut $\mathrm{LiNbO}_{3}$ with different metallization ratios. The sensitivity increases when the metallization ratio increases from 0.4 to 0.6 .

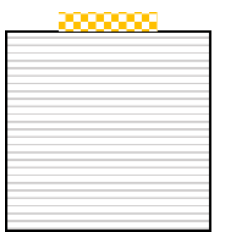

Ferromagnetic electrode

Substrate

Fig. 3. The diagram of the ferromagnetic electrodes/substrate structure. The ferromagnetic electrodes are deposited on the piezoelectric substrate.

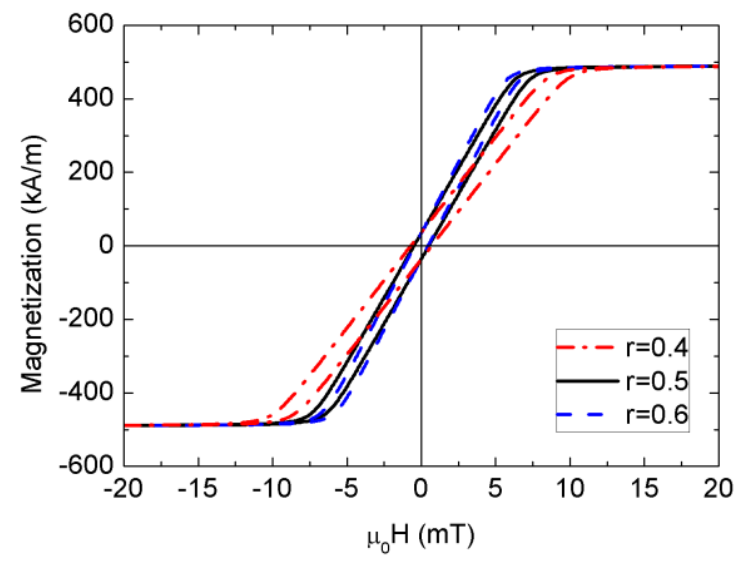

Fig. 4. Calculated magnetizations of $\mathrm{Ni}$ electrodes with different metallization ratios ( $r$ ) along the hard axis.

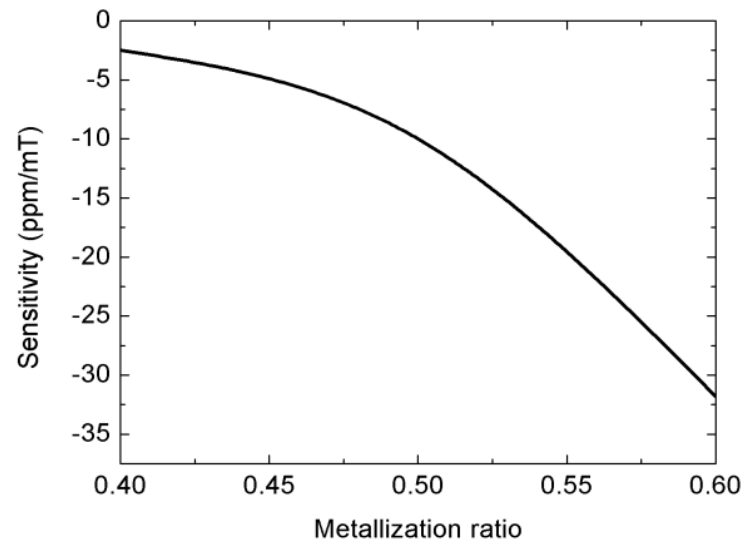

Fig. 5. Calculated sensitivities of Rayleigh wave mode on $\mathrm{Ni}$ electrodes $/ 41^{\circ} \mathrm{YX}$-cut $\mathrm{LiNbO}_{3}$ with different metallization ratios.

The calculated frequency shifts of the Rayleigh wave mode versus magnetic fields are illustrated in Fig. 6, compared with experimental results adapted from Ref. [23]. The metallization ratio is 0.5 , and the saturation magnetic flux density is set as $8 \mathrm{mT}$. The contribution to the frequencymagnetic field dependence from $\Delta \mathrm{E}$ effect and the interaction of magnetostriction effect and SAW are compared. The Rayleigh wave mode is mainly sensitive to the $c_{11}$ variations of the ferromagnetic electrodes or films. If only the $\Delta \mathrm{E}$ effect sequential coupling is considered in the model, the curve shape is similar to the experiment, but the calculated frequency shifts will be far from the experimental results when the magnetic field increases above the saturation magnetic flux density, and the difference is noticeable. The 
simulation result of the multi-physics coupling model is in a good agreement with the experimental data. Because of the negative magnetostrictive coefficient of $\mathrm{Ni}$, the frequency variations are closer to the experiment data by taking account of interactions among magnetostrictive strains, stresses, and SAW. The agreement between the calculation and experimental data confirms the importance of importing the magnetostriction effect into the calculation.

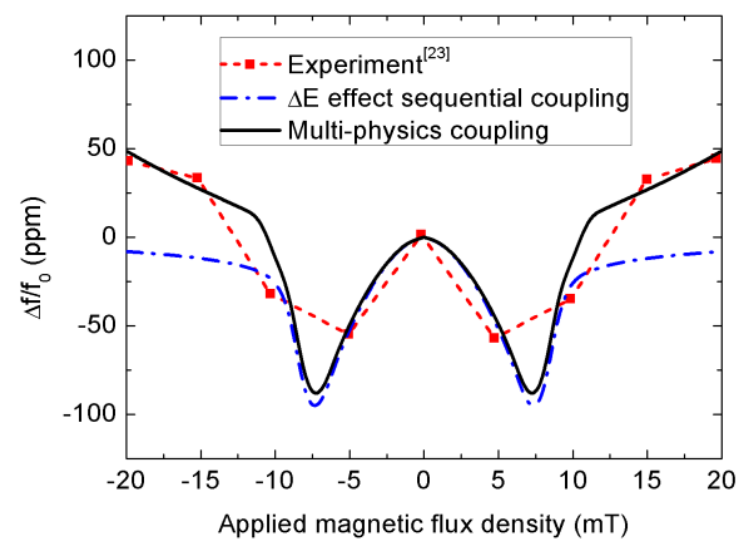

Fig. 6. The calculated relative frequency shifts of Rayleigh wave mode on $\mathrm{Ni} / 41^{\circ} \mathrm{YX}$-cut $\mathrm{LiNbO}_{3}$ as a function of the applied magnetic flux density when the magnetic field is parallel to the hard axis.

\section{2) ( $\mathrm{TbCo} / \mathrm{FeCo}$ multi-layer) $/ 128^{\circ} \mathrm{YX}$-cut $\mathrm{LiNbO}_{3}$ structure}

The magnetic field sensitivity of nanostructured magnetostrictive $\mathrm{TbCo}_{2} / \mathrm{FeCo}$ multi-layered electrodes $/ 128^{\circ}$ YX-cut $\mathrm{LiNbO}_{3}$ resonator is also calculated for studying the sensing mechanism and model validation. The shapes of the $\mathrm{TbCo}_{2} / \mathrm{FeCo}$ multi-layer electrodes are also set as cuboids in the multi-physics coupling model. The thicknesses of $\mathrm{TbCo}_{2} / \mathrm{FeCo}$ multi-layered electrodes are $200 \mathrm{~nm}$. The wavelength is $6.5 \mu \mathrm{m}$, and the metallization ratio is 0.6 . The material constants of $\mathrm{TbCo}_{2} / \mathrm{FeCo}$ are as follows: $\rho_{0}=9210$ $\mathrm{kg} / \mathrm{m}^{3}, c_{11}=113.7 \mathrm{GPa}, c_{12}=51.1 \mathrm{GPa}, c_{44}=31.3 \mathrm{GPa}$, and $M_{s}=$ $800 \mathrm{kA} / \mathrm{m}$ [17]. The coercive field is $5.95 \times 10^{3} \mathrm{~A} / \mathrm{m}$. The hard axis is parallel to the SAW propagation direction. The saturation magnetic flux density is close to $200 \mathrm{mT}$ along the hard axis when the magnetization reaches the saturation. Although $\mathrm{TbCo}_{2} / \mathrm{FeCo}$ is magnetically harder than $\mathrm{Ni}$ electrodes, the shapes of $\mathrm{TbCo}_{2} / \mathrm{FeCo}$ electrodes still have an impact on anisotropy and magnetization, as shown in Fig. 7.

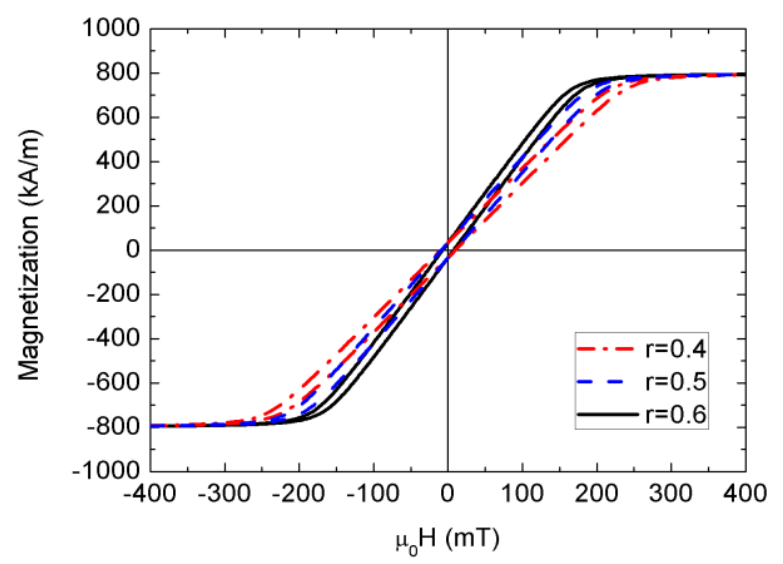

Fig. 7. Calculated magnetizations of $\mathrm{TbCo}_{2} / \mathrm{FeCo}$ electrodes with different metallization ratios ( $\mathrm{r}$ ) along the hard axis.

Fig. 8 illustrates the simulated relative frequency variations of the Rayleigh wave mode as a function of the applied magnetic field that is parallel to the hard axis, compared with the experimental data adapted from Ref. [24]. $\Delta \mathrm{E}$ effect is the major sensitive effect in $\mathrm{TbCo}_{2} / \mathrm{FeCo}$. The turnover point is around the saturation magnetic flux density. The magnetic field sensitivity is $-0.27 \mathrm{ppm} / \mathrm{mT}$ from 0 to 200 $\mathrm{mT}$. The resonance frequency decreases to the minimum at $200 \mathrm{mT}$. Then, the frequency increases to a saturation value when the magnetic field is above $200 \mathrm{mT}$. It is found that the increased saturation magnetic flux density is counterproductive for the magnetic sensitivity. The agreement between the simulation and experiment confirms the accuracy of the SAW multi-physics coupling model.

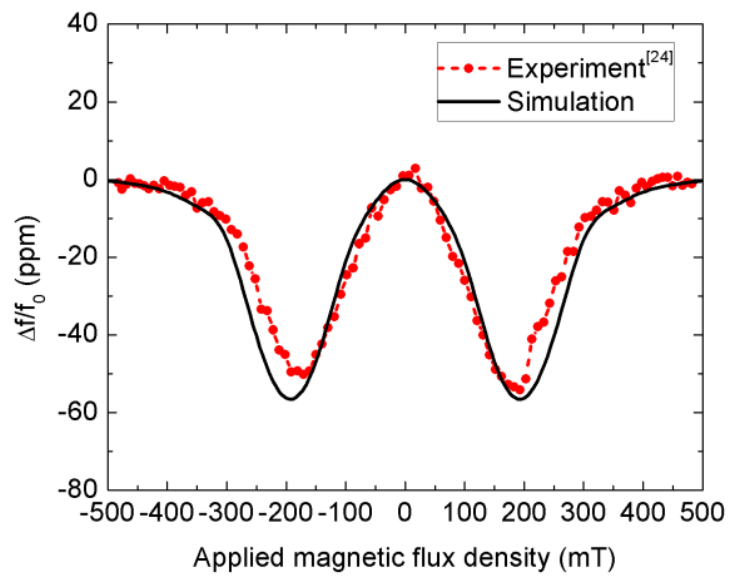

Fig. 8. The calculated relative frequency shifts of Rayleigh wave mode on $\left(\mathrm{TbCo}_{2} / \mathrm{FeCo}\right) / 128^{\circ} \mathrm{YX}$-cut $\mathrm{LiNbO}_{3}$ as a function of an external magnetic field applied along the hard axis.

The shape demagnetizing factor distributions in ferromagnetic electrodes are illustrated in Fig. 9. The wavelength is $6.5 \mu \mathrm{m}$. The symbols $\mathrm{r}$ and $\mathrm{h}$ represent the metallization ratio and the thickness of the ferromagnetic electrode, respectively. The aperture length is set as $500 \mu \mathrm{m}$. The shape demagnetizing factors of ferromagnetic electrodes in the $\mathrm{x}$ direction are $10^{3}$ times larger than those of a ferromagnetic film in Fig. 2(a). Compared with a 
ferromagnetic film, the shape demagnetizing effect is significant in ferromagnetic electrodes due to the narrow widths of electrodes.

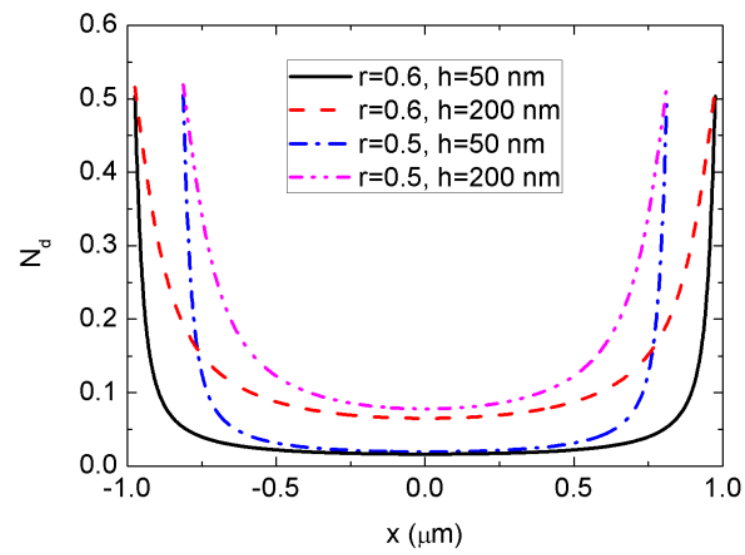

Fig. 9. Shape demagnetization factor distributions of ferromagnetic electrodes in the $\mathrm{x}$ direction.

\section{B. Ferromagnetic film as a layer}

In this section, the approaches for improved sensitivities are explored. In the above ferromagnetic electrodes/substrate structure presented here, only a part of SAW traverses in the ferromagnetic electrodes deposited on the substrate. As an alternative, a ferromagnetic full film can be also used as an active sensing layer, as shown in Fig. 10. The isolating layer is added between the ferromagnetic film and electrodes. This structure provides larger sensing areas compared to the previous one mentioned above. Therefore, a ferromagnetic film/isolating layer/substrate multi-layered structure is studied. The isolating layer protects electrodes naturally. The ferromagnetic film is deposited on the top surface of the isolating layer to enhance the sensing areas and thus improve the sensitivity.

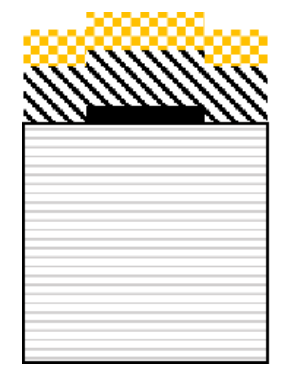

Ferromagnetic film

Isolating layer

Electrode

\section{Substrate}

Fig. 10. The schematic diagram of the ferromagnetic film/isolating layer/substrate multi-layered structure.

\section{1) $\mathrm{Ni} / \mathrm{ZnO} / 128^{\circ} \mathrm{YX}$-cut $\mathrm{LiNbO}_{3}$ structure}

The frequency variations with the applied magnetic field for the Rayleigh wave mode on the $\mathrm{Ni} / \mathrm{ZnO} / 128^{\circ} \mathrm{YX}$-cut $\mathrm{LiNbO}_{3}$ are simulated, compared with experimental data adapted from Ref. [25], and illustrated in Fig. 11. ZnO thin film is used as the isolating layer. The wavelength is $24 \mu \mathrm{m}$. The $\mathrm{ZnO}$ thickness is $250 \mathrm{~nm}$, and the Ni film thickness is 200 $\mathrm{nm}$. The calculations match well with the experimental curves. The model is validated in this multi-layered structure. Compared with the $\mathrm{Ni}$ electrodes in Fig. 6, the $\mathrm{Ni}$ film becomes magnetically harder. The minima point shifts to 150
mT using a full film instead of using $\mathrm{Ni}$ as electrodes. This is because the Ni full film has a much larger saturation magnetic flux density than the $\mathrm{Ni}$ electrodes. The saturation magnetic flux density is the main factor that decides the magnetic field where the minima is achieved. The saturation magnetic flux density of the $200 \mathrm{~nm} \mathrm{Ni}$ full film is around $150 \mathrm{mT}$ in the hard axis. The sensitivity of the fundamental mode is -0.4 $\mathrm{ppm} / \mathrm{mT}$ using Ni as a full film lower than that of $-10 \mathrm{ppm} / \mathrm{mT}$ using $\mathrm{Ni}$ as electrodes in Fig. 6.

In Fig. 11, The magnetic field sensitivity of the third harmonic Rayleigh wave mode is $-1.32 \mathrm{ppm} / \mathrm{mT}$ that is three times as large as that of the fundamental mode. This is because the wave energy of the third harmonic mode is more concentrated on the top surface than that of the fundamental mode. The displacement distributions are shown in Fig. 12, it is quite clear that the energy of the fundamental mode and the third harmonic mode are mostly concentrated in the top surface. Besides, compared with the fundamental mode, the third harmonic mode enables much more energy trapping in the ferromagnetic layer and thus offers a larger sensitivity. Therefore, more SAW energy in the ferromagnetic film is beneficial for a larger magnetic field sensitivity.

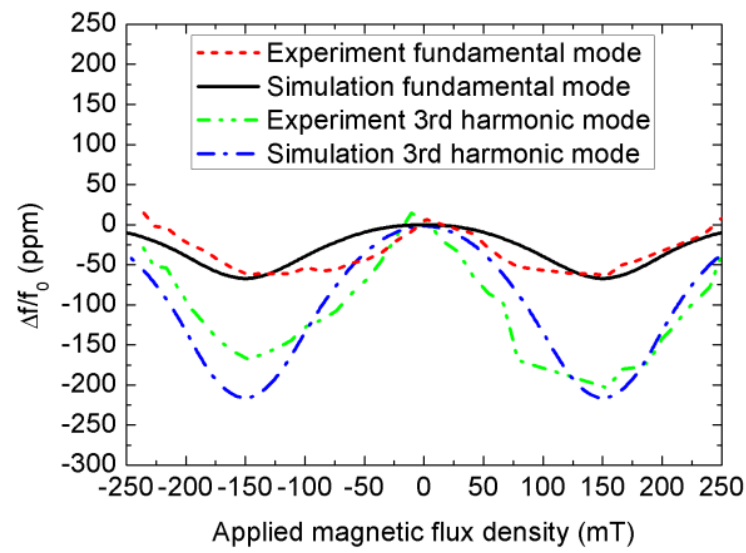

Fig. 11. The calculated relative frequency shifts of Rayleigh wave mode on $\mathrm{Ni} / \mathrm{ZnO} / 128^{\circ} \mathrm{YX}$-cut $\mathrm{LiNbO}_{3}$ as a function of an external magnetic field applied along the hard axis.

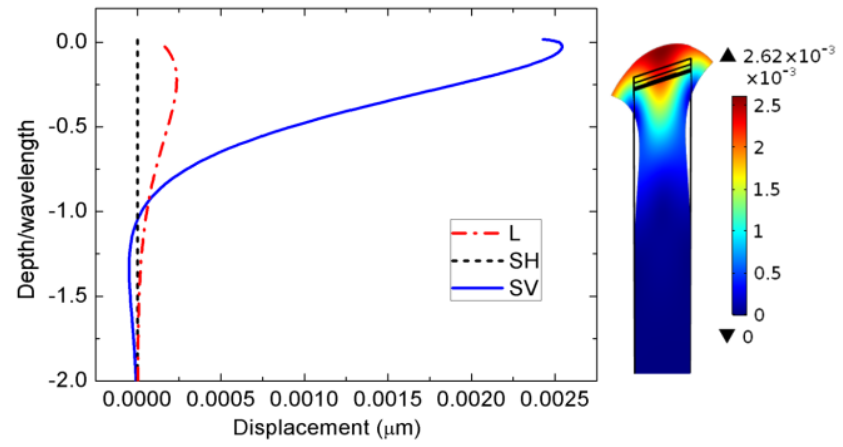

(a) 


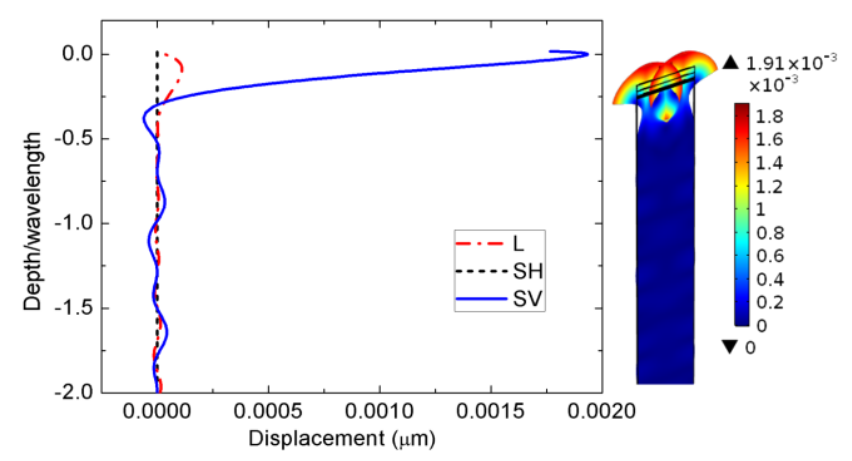

(b)

Fig. 12. Displacement distributions of Rayleigh wave along the depth of $\mathrm{Ni} / \mathrm{ZnO} / 128^{\circ} \mathrm{YX}$-cut $\mathrm{LiNbO}_{3}$ structure at the resonance frequency for (a) the fundamental Rayleigh wave mode. (b) the third harmonic Rayleigh wave mode.

\section{2) $\mathrm{CoFeB} / \mathrm{ZnO} / 128^{\circ} \mathrm{YX}$-cut $\mathrm{LiNbO}_{3}$ structure}

$\mathrm{CoFeB}$ is one of soft ferromagnetic materials that have a near zero magnetic remanence and a negligible coercive field $80 \mathrm{~A} / \mathrm{m}$ that is much lower than that of $\mathrm{TbCo}_{2} / \mathrm{FeCo}$. Hence the hysteresis is negligible for $\mathrm{CoFeB}$ films. The material constants of $\mathrm{CoFeB}$ used for a model are as follows: Young's modulus $=160 \mathrm{GPa}$, Poisson's ratio=0.37, $M_{s}=954.9 \mathrm{kA} / \mathrm{m}$ [26]. It has a lower saturation magnetic flux density around $5 \mathrm{mT}$ along the hard axis than that of $\mathrm{TbCo}_{2} / \mathrm{FeCo}$ that allows to get a higher sensitivity. Therefore, $\mathrm{CoFeB}$ is an ideal material for improving sensitivities of SAW magnetic field sensors.

The calculated magnetizations of $\mathrm{CoFeB}$ films with different shape demagnetization factors along the wave propagation direction are shown in Fig. 13. As the shape demagnetization factor increases from $10^{-5}$ to $10^{-4}$, the magnetization curves and the anisotropy field change little. Although the shape demagnetization factor affects the anisotropy as it increases to $10^{-3}$, the anisotropy field and saturation magnetic flux density shift slightly. Because the shape demagnetization factors are nearly close to 0 in soft magnetic films, the shape demagnetizing field is negligible and therefore has only a minor impact on the sensitivity. It is one of the advantages of the full ferromagnetic film configuration.

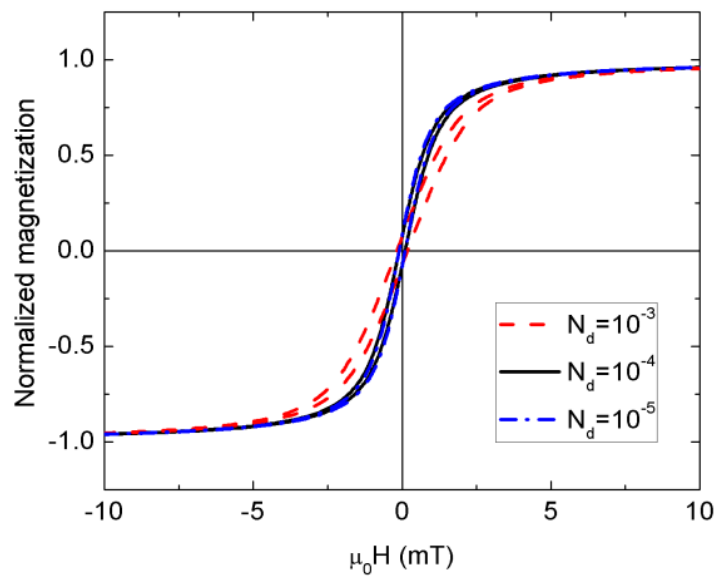

Fig. 13. Calculated magnetizations of $\mathrm{CoFeB}$ films with different shape demagnetization factors $\mathrm{N}_{\mathrm{d}}$ in the $\mathrm{x}$ direction.
The magnetic field sensitivity of the Rayleigh wave mode on the $\mathrm{CoFeB} / \mathrm{ZnO} / 128^{\circ} \mathrm{YX}$-cut $\mathrm{LiNbO}_{3}$ structure is calculated. The wavelength is $24 \mu \mathrm{m}$. The $\mathrm{ZnO}$ thickness is $250 \mathrm{~nm}$, and the CoFeB film thickness is $200 \mathrm{~nm}$. The calculated frequency variations versus the applied magnetic field are illustrated in Fig. 14 compared with experimental results adapted from Ref. [27]. There is a relatively agreement between the experimental and simulated results. The relative frequency variation decreases to the minimum $-67 \mathrm{ppm}$ when the magnetic field increases from 0 to $5 \mathrm{mT}$. The magnetic sensitivity is $-13.4 \mathrm{ppm} / \mathrm{mT}$ in this magnetic field range. It is found that the lower saturation magnetic flux density is beneficial to obtain the higher magnetic field sensitivity.

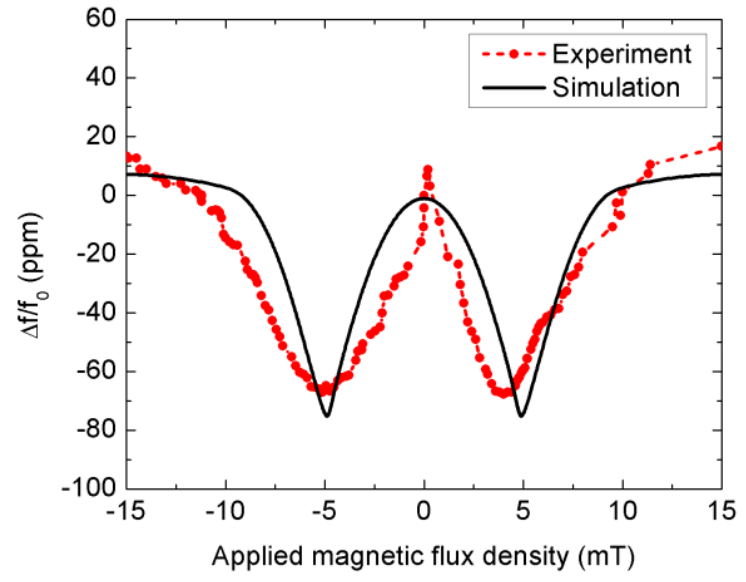

Fig. 14. The calculated relative frequency shifts of Rayleigh wave mode on $\mathrm{CoFeB} / \mathrm{ZnO} / 128^{\circ} \mathrm{YX}$-cut $\mathrm{LiNbO}_{3}$ versus applied magnetic fields.

3) Love wave mode on a grooved sensing surface

As mentioned above, more confinement of SAW energy on the surface is beneficial for larger sensitivity. Love wave mode is more suitable for this sensing mechanism, because the acoustic velocity difference between layers favors the concentration of wave energy into a thin ferromagnetic film. It is also beneficial to the magnetic sensitivity of Love waves due to the fact that they depend heavily on the variations of the shear modulus that tends to be larger than that of the Young modulus [28].

A grooved ferromagnetic film sensing surface based on ST-quartz used in the $\mathrm{X}+90^{\circ}$ direction is proposed for a high sensitivity using the Love wave mode, as shown in Fig. 15. $\mathrm{ZnO}$ thickness is $300 \mathrm{~nm}$, and the $\mathrm{SiO}_{2}$ is placed on the polished flat upper surface of the $\mathrm{ZnO}$ layer. The $\mathrm{SiO}_{2}$ thickness is $250 \mathrm{~nm}$. The upper surface of the $\mathrm{SiO}_{2}$ layer is grooved. $\mathrm{CoFeB}$ is placed over the grooves of the $\mathrm{SiO}_{2}$ layer. The upper surface of the $\mathrm{CoFeB}$ film is considered as polished to be kept flat. The magnetostrictive displacement deformations of grooved sensing surface structures in the external applied magnetic field are shown in Fig. 16. Compared with the flat film structure, grooved structures enhance the sensing areas and magnetostrictive displacement deformations. Moreover, the value of the maximum displacement at the groove sensing surface increases as the depth of groove expands from $50 \mathrm{~nm}$ to $150 \mathrm{~nm}$. Effective magnetic field intensity distribution regions are also increased 
in grooved sensing surface structures, as shown in Fig. 17. Fig. 18 illustrates the resonance frequency variations of Love waves with different depths of the grooves. As the depth of the groove is $50 \mathrm{~nm}$, the maximum frequency variation is $2102 \mathrm{ppm}$. As the depth of the groove is $100 \mathrm{~nm}$, the maximum frequency variation is $-2664 \mathrm{ppm}$, and the magnetic field sensitivity is $-532.8 \mathrm{ppm} / \mathrm{mT}$ from 0 to $5 \mathrm{mT}$. When the depth of the groove increases to $150 \mathrm{~nm}$, the maximum frequency shift is - $3208 \mathrm{ppm}$, the magnetic field sensitivity is $-641.6 \mathrm{ppm} / \mathrm{mT}$, and the magnetic field resolution is 3.6 $\mu \mathrm{T} / \mathrm{kHz}$.

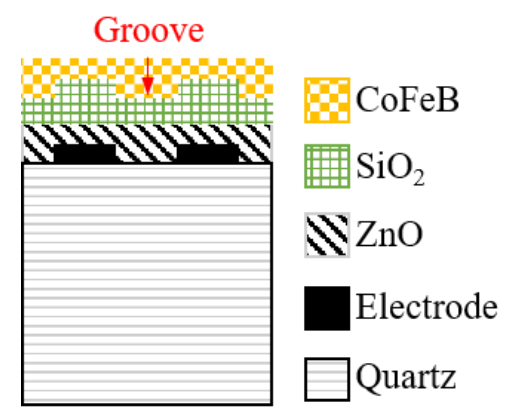

Fig. 15. The schematic diagram of a grooved sensing surface structure.

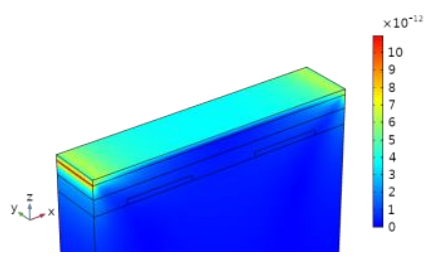

(a)

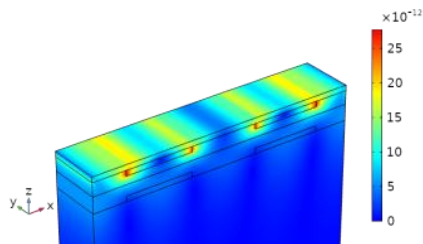

(c)

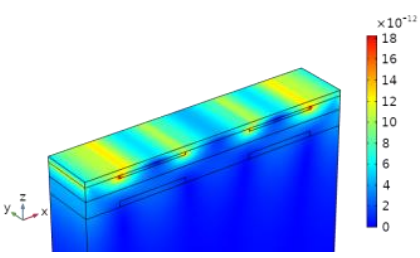

(b)

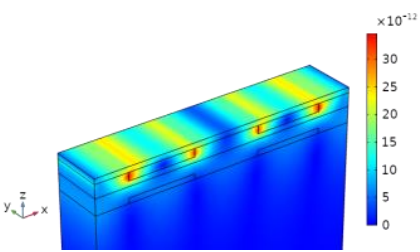

(d)
Fig. 16. Magnetostrictive displacement deformations of a flat film structure and grooved sensing surface structures (a) a flat film structure. (b) the depth of the groove is $50 \mathrm{~nm}$. (c) the depth of the groove is $100 \mathrm{~nm}$. (d) the depth of the groove is $150 \mathrm{~nm}$.

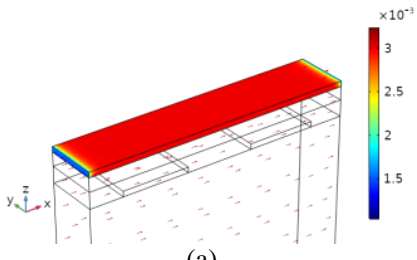

(a)

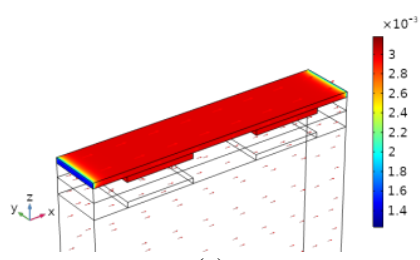

(c)

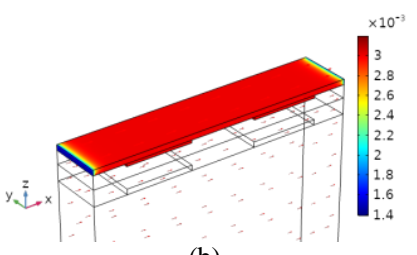

(b)

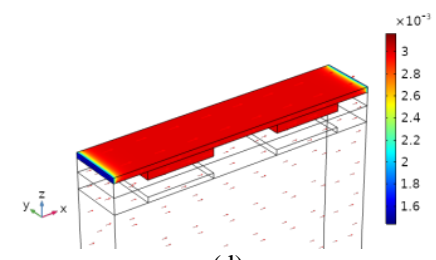

(d)
Fig. 17. Effective magnetic field intensity distributions of a flat film structure and grooved sensing surface structures (a) a flat film structure. (b) the depth of the groove is $50 \mathrm{~nm}$. (c) the depth of the groove is $100 \mathrm{~nm}$. (d) the depth of the groove is $150 \mathrm{~nm}$.

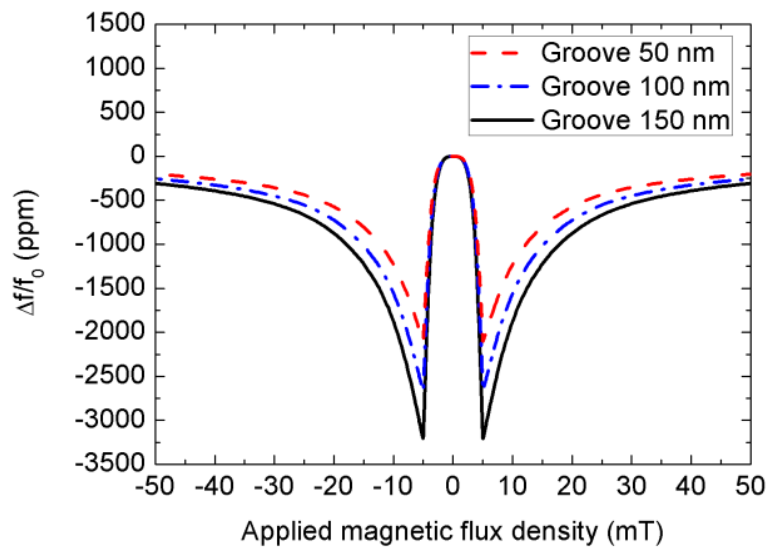

Fig. 18. Calculated relative frequency variations of the Love wave mode on the grooved $\mathrm{CoFeB} / \mathrm{SiO}_{2} / \mathrm{ZnO} / \mathrm{ST}+90^{\circ}$ - cut quartz as a function of an external magnetic field applied along the hard axis.

\section{CONCLUSION}

The sensing mechanism of SAW magnetic field sensors is studied by the SAW multi-physics coupling model. The magnetostriction effect, $\Delta \mathrm{E}$ effect, and nonlinear magnetizations with demagnetizing effect are taken into consideration for the interaction of magnetic field and SAW propagation. The model provides a good agreement between the simulation results and experimental results from the literature. It is found that the more SAW energy on the top surface helps obtain an improved sensitivity. A ferromagnetic film with a reduced saturation magnetic flux density is also beneficial for improving the sensor's sensitivity. Increasing the length-width ratio and length-height ratio of a ferromagnetic film decreases the demagnetizing field. A grooved sensing surface structure is proposed and exhibits a higher theoretical sensitivity. The results are promising for the design of one-port SAW magnetic field resonators as wireless and passive magnetic field sensors.

\section{REFERENCES}

[1] P. Ripka, M. Janosek. "Advances in Magnetic Field Sensors." IEEE Sensors Journal, vol. 10, no. 6, pp. 1108-1116, 2010.

[2] J. Lenz, A. S. Edelstein. "Magnetic sensors and their applications." IEEE Sensors Journal, vol. 6, no. 3, pp. 631-649, 2006.

[3] G. Scholl, F. Schmidt, T. Ostertag, and L. Reindl, "Wireless passive SAW sensor systems for industrial and domestic applications," Proceedings of the 1998 IEEE International Frequency Control Symposium, pp. 595-601, 1998.

[4] M. J. Dapino. "On magnetostrictive materials and their use in adaptive structures." Structural Engineering and Mechanics, vol. 17, no. 3-4, pp. 303-330, 2004.

[5] W. P. Robbins, A. Young. "SAW phase modulator using magnetostrictive thin films." IEEE transactions on sonics and ultrasonics, vol. 32, no. 3, pp. 423-427, 1985.

[6] H. Mishra, J. Streque, M. Hehn, et al. "Temperature compensated magnetic field sensor based on love waves." Smart Materials and Structures, vol. 29, no. 4, pp. 045036: 1-6, March. 2020.

[7] W. Wang, Y. Jia, X. Liu, et al. "Performance improvement of the SAW based current sensor incorporating a strip-patterned magnetostrictive FeCo film.” 2017 IEEE International Ultrasonics Symposium (IUS). IEEE, pp. 1-3, 2017. 
[8] M. Kadota, S. Ito. "Sensitivity of surface acoustic wave magnetic sensors composed of various Ni electrode structures." Japanese Journal of Applied Physics, vol. 51, no. 7S, pp. 07GC21-1-5, 2012.

[9] M. Yamaguchi, K. Hashimoto, H. Kogo, et al. "Variable SAW delay line using amorphous $\mathrm{TbFe}_{2}$ film." IEEE Transactions on Magnetics, vol. 16, no. 5, pp. 916-918, 1980.

[10] K. Hashimoto, M. Yamaguchi, H. Kogo, et al. "Magnetostrictive properties of sputtered Co-Cr film on surface acoustic wave." IEEE Transactions on Magnetics, vol. 17, no. 6, pp. 3181-3183, 1981.

[11] W. Wang, Y. Jia, X. Xue, et al. "Grating-patterned FeCo coated surface acoustic wave device for sensing magnetic field." Aip Advances, vol. 8, no. 1, pp. 015134, 2018.

[12] W. Li, P. Dhagat, A. Jander. "Surface acoustic wave magnetic sensor using galfenol thin film." IEEE transactions on magnetics, vol. 48, no. 11, pp. 4100-4102, 2012.

[13] J. Labrenz, A. Bahr, P. Durdaut, et al., "Frequency Response of SAW Delay Line Magnetic Field/Current Sensor," IEEE Sensors Letters, vol. 3, no. 10, pp. 1-4, Art no. 1500404, 2019.

[14] B. K. Sinha, H. F. Tiersten, "On the temperature dependence of the velocity of surface waves in quartz," J. Appl. Phys., vol. 51, no. 1, pp. 4659-4665, 1980.

[15] H. F. Tiersten, "Perturbation theory for linear electroelastic equations for small fields superposed on a bias," J. Acoust. Soc. Am. vol. 64, no. 3, pp. 832-837, 1978.

[16] Y. Yang, H. Mishra, Q. Z. Zhang, S. Hage-Ali, T. Han, O. Elmazria, "A Weak Form Nonlinear Model for Thermal Sensitivity of Love Wave Mode on Layered Structures." IEEE Transactions on Ultrasonics, Ferroelectrics, and Frequency Control, vol. 67, no. 6, pp. 1275-1283, 2020.

[17] H. Zhou, A. Talbi, N. Tiercelin, et al. "Multilayer magnetostrictive structure based surface acoustic wave devices." Applied Physics Letters, vol. 104, no. 11, pp. 114101, 2014

[18] Y. Yang, H. Mishra, P. Mengue, et al. "Enhanced Performance Love Wave Magnetic Field Sensors with Temperature Compensation," IEEE Sensors Journal, 2020, 20(19): 11292-11301.

[19] D. C. Jiles. "Theory of the magnetomechanical effect." Journal of physics D: Applied Physics, vol. 28, no. 8, pp. 1537, 1995.

[20] R. I. Joseph, E. Schloimann. "Demagnetizing Field in Nonellipsoidal Bodies." Journal of Applied Physics, vol. 36, no. 5, pp.1579-159, 1965.

[21] Y. Cho, K. Yamanouchi. "Nonlinear, elastic, piezoelectric, electrostrictive, and dielectric constants of lithium niobate." Journal of applied physics, vol. 61, no. 3, pp. 875-887, 1987.

[22] V. P. N. Sarma, P. J. Reddy. "Measurement of third-order elastic constants of single crystal nickel at $298^{\circ}$ K." Philosophical Magazine, vol. 27, no. 4, pp. 769-775, 1973.

[23] V. Polewczyk, K. Dumesnil, D. Lacour, et al. "Unipolar and bipolar high-magnetic-field sensors based on surface acoustic wave resonators." Physical Review Applied, vol. 8, no. 2, pp. 024001, 2017.

[24] H. Mishra, M. Hehn, D. Lacour, et al. "Intrinsic versus shape anisotropy in micro-structured magnetostrictive thin films for magnetic surface acoustic wave sensors." Smart Materials and Structures, vol. 28, no. 12, pp. 12LT01:1-6, November. 2019.

[25] M. Elhosni, O. Elmazria, S. Petit-Watelot, et al. "Magnetic field SAW sensors based on magnetostrictive-piezoelectric layered structures: FEM modeling and experimental validation." Sensors and Actuators A: Physical, vol. 240, pp. 41-49, 2016.

[26] M. Gueye, F. Zighem, M. Belmeguenai, M. S. Gabor, C. Tiusan, and D Faurie, "Spectroscopic investigation of elastic and magnetoelastic properties of CoFeB thin films." Journal of Physics D: Applied Physics, vol. 49, no.14, pp. 145003, 2016.

[27] M. Elhosni, Petit-Watelot, Sébastien, M. Hehn, et al. "Experimental Study of Multilayer Piezo-magnetic SAW Delay Line for Magnetic Sensor." Procedia Engineering, vol. 120, pp. 870-873, 2015.

[28] A. Kittmann, P. Durdaut, S. Zabel, J. Reermann, J. Schmalz, B. Spetzler, D. Meyners, N. X. Sun, J. McCord, M. Gerken, G. Schmidt, M. Höft, R. Knöchel, F. Faupel and E. Quandt, "Wide Band Low Noise Love Wave Magnetic Field Sensor System," Scientific Reports, vol. 8, no. 1, pp. $278-285,2018$

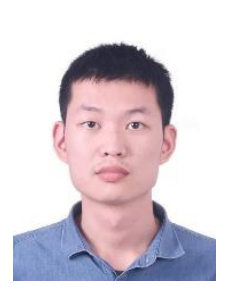

Yang Yang was born in Jiangsu, China. He received the bachelor degree from University of Electronic Science and Technology of China in 2015. He is currently a Ph.D. student at School of Electronic Information and Electrical Engineering in Shanghai Jiao Tong University. His current research interests include wireless surface acoustic wave sensors and signal processing.

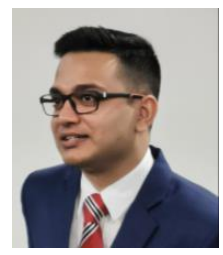

Harshad Mishra was born in Odisha, India, in 1989. He received his Ph.D. degree from Institut Jean Lamour, Université de Lorraine, France in 2019. Prior to that, he received his M.S. degree from IIT Madras, India in 2016. $\mathrm{He}$ is currently a post-doctoral researcher at Aalto University, Finland. His research interests include the development and optimization of magnetic field sensors using micro-structured magnetoelastic thin films, devices based on surface acoustic waves, nanofabrication technologies and exploring the interactions between elastic waves and magnons at the mechanical quantum limit.

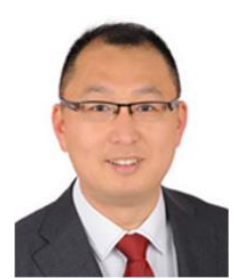

Tao Han was born in Shandong, China, in 1973. He received the Ph.D. degree in instrument science and technology from Shanghai Jiao Tong University, Shanghai, China, in 2002. He was a Visiting Scholar with Tohoku University, Sendai, Japan, in 2003. He is currently a professor at School of Electronic Information and Electrical Engineering in Shanghai Jiao Tong University. His current research interests include acoustic wave devices simulation, wireless surface acoustic wave sensors system, and ultrasound-based measurement. Prof. Han is a Technical Program Committee Member of the IEEE Ultrasonics Symposium.

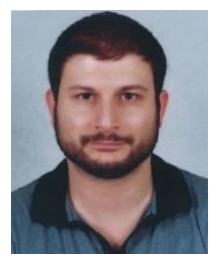

Sami Hage-Ali was born in Strasbourg, France, in 1982. He received an Engineering Degree from Ecole Centrale de Lille and a M.S in micro-nanotechnology from University of Lille 1 in 2005. He received another Master's Degree in international projects engineering from University of Lille 1 in 2006. He received a Ph.D. in micro-nanotechnology, acoustics and telecommunications from Ecole Centrale de Lille in 2011. In 2011, Dr. Hage-Ali was awarded a Fulbright grant and became a post-doctoral fellow at the University of Illinois at Urbana-Champaign, USA. Since 2014, he is an Associate professor at Universite de Lorraine and is with the Micro-nanosystems group of Institut Jean Lamour, Nancy, France. His research interests include surface acoustic wave 
sensors, flexible/stretchable electronics, micro-nanosystems, microwaves, and antennas. He is the founder of the IEEE France Section Sensors Council Chapter.

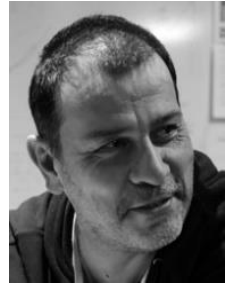

Michel Hehn received the Ph.D. degree from the University of Strasbourg in 1996, joined the French National Center of Scientific Research in 1998 and became a professor at University de Lorraine in 2006. $\mathrm{He}$ is also a member of the IUF (Institut Universitaire de France). He is a specialist in material growth and in nanomagnetism/spintronics. In 2010, he won the Yves Rocard 2010 Price of the French Society of Physics for the invention and the technological development of "a new generation of magnetic sensors for ASB" for the SNR society. He has coauthored more than 270 papers in refereed international journals and in proceeding of international conferences.

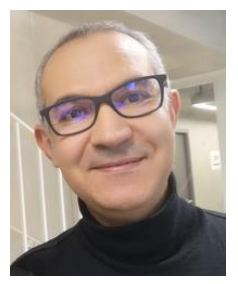

Omar Elmazria was born in Casablanca, Morocco in 1968. He received his Ph.D. degree in Electronic from University of Metz, France. $\mathrm{He}$ is currently a Full Professor at Université de Lorraine, France and the Head of the Micro and Nanosystems Group within the Institut Jean Lamour, Nancy, France. He is also director for international affairs at Polytech Nancy, Franc, and was worldwide guest Professor (Simon Fraser University, BC, Canada; Institute of Acoustic, Chinese Academy of Sciences, Beijing, China; and University of Central Florida, FL, USA). His current research focuses on SAW devices for communication systems and sensing applications. He has co-authored more than 200 papers in refereed international journals and in proceeding of international conferences. Prof. Elmazria is a Technical Program Committee Member of the IEEE IUS and IEEE MTT - 26. In 2017, he was a recipient of the URSI-France medal from the International Union of Radio Science. 